\title{
Global Convexity in the Bi-Criteria Traveling Salesman Problem
}

\author{
Marcos Villagra ${ }^{1,2}$, Benjamín Barán ${ }^{1,2}$, and Osvaldo Gómez ${ }^{1,2}$ \\ 1 National University of Asuncion \\ 2 Catholic University of Asuncion \\ \{mvillagra, bbaran\}@cnc.una.py, ogomez@illigal.ge.uiuc.edu
}

\begin{abstract}
This work studies the solution space topology of the Traveling Salesman Problem or TSP, as a bi-objective optimization problem. The concepts of category and range of a solution are introduced for the first time in this analysis. These concepts relate each solution of a population to a Pareto set, presenting a more rigorous theoretical framework than previous works studying global convexity for the multi-objective TSP. The conjecture of a globally convex structure for the solution space of the bi-criteria TSP is confirmed with the results presented in this work. This may support successful applications using state of the art metaheuristics based on Ant Colony or Evolutionary Computation.
\end{abstract}

Key words: Traveling Salesman Problem, Multi-Objective Optimization, Global Convexity.

\section{Introduction}

Metaheuristics are a class of optimization algorithms that today constitute one of the best options to solve very complex problems. These algorithms try to combine basic heuristic methods in higher level frameworks aimed at efficiently and effectively exploring a search space [1].

The research in the field of metaheuristics has evolved on the basis of trial and error [2], often motivated by the competition for improving the best known solutions for given problems, and not by identifying the reasons for the success and failure of these algorithms.

The Traveling Salesman Problem or TSP has been used as a benchmark problem for the study of many metaheuristics. The topology of the singleobjective TSP has been study in [3-6], and the three-objective TSP in [2], for specific instances. In general, all these results suggest that the solution space has a globally convex structure.

Global Convexity is not convexity in the strict sense [2], but may be used to denote the empirical observation that the best local optima are gathered in a small part of the solution space, which hopefully includes the global optimum. Metaheuristics exploit this by concentrating their search in that part of the solution space [2].

Please use the following format when citing this chapter:

Villagra, M., Barán, B., Gómez, O., 2006, in IFIP International Federation for Information Processing, Volume 217, Artificial Intelligence in Theory and Practice, ed. M. Bramer, (Boston: Springer), pp. 217-226. 
This work studies the solution space topology of the bi-objective TSP in a more practical way than the studies carried out in [2], by means of two new metrics, category and range of a solution. The former, relates a solution with the number of solutions that dominate it; the latter, establishes a hierarchy in the solution space. The whole solution space was studied for random instances with 7,8,9 and 10 cities. Then, subsets of the solution space ware analyzed for larger problems with 100 and 150 cities. It is interesting to mention that when global convexity exists, it may be exploited in metaheuristics for multiobjective combinatorial optimization [2]. Global convexity can be used to design good algorithms or to explain the reason of success of well known metaheuristics that make good use of this property, like Ant Colony Optimization (ACO) and Evolutionary Algorithms (EA) [7].

The remainder of this work is organized as follows. Section 2 presents a general definition of a multiple objective problem. The multi-objective TSP is presented in section 3. Global convexity is described in section 4 . The theoretical framework and experimental results are explained in section 5. Finally, conclusions and future work are left for section 6 .

\section{Multi-Objective Optimization Problems}

A general Multi-Objective Optimization Problem (MOP) includes a set of $n$ decision variables, $k$ objective functions, and $m$ restrictions. Objective functions and restrictions are functions of decision variables. This can be expressed as:

$$
\begin{aligned}
& \text { Optimize } \mathbf{y}=f(\mathbf{x})=\left(f_{1}(\mathbf{x}), f_{2}(\mathbf{x}), \ldots, f_{k}(\mathbf{x})\right) \\
& \text { Subject to } \gamma(\mathbf{x})=\left(\gamma_{1}(\mathbf{x}), \ldots, \gamma_{m}(\mathbf{x})\right) \geq 0 \\
& \text { where } \quad \mathbf{x}=\left(x_{1}, x_{2}, \ldots, x_{n}\right) \in \mathbf{X} \text { is the decision vector, and } \\
& \qquad \mathbf{y}=\left(y_{1}, y_{2}, \ldots, y_{k}\right) \in \mathbf{Y} \text { is the objective vector }
\end{aligned}
$$

$\mathbf{X}$ denotes the decision space while $\mathbf{Y}$ is the objective space. Depending on the problem, "optimize" could mean minimize or maximize. The set of restrictions $\gamma(\mathbf{x}) \geq 0$ determines the set of feasible solutions $\mathbf{X}_{f} \subseteq \mathbf{X}$ and its corresponding set of objective vectors $\mathbf{Y}_{f} \subseteq \mathbf{Y}$. A multi-objective problem consists in finding $\mathbf{x}$ that optimizes $f(\mathbf{x})$. In general, there is no unique "best" solution but a set of solutions, none of which can be considered better than the others when all objectives are considered at the same time. This comes from the fact that there can be conflicting objectives. Thus, a new concept of optimality should be established for MOPs. Given two decision vectors $\mathbf{u}, \mathbf{v} \in X$ :

$$
\begin{array}{llll}
f(\mathbf{u})=f(\mathbf{v}) & \text { iff } & \forall i \in 1,2, \ldots, k: & f_{i}(\mathbf{u})=f_{i}(\mathbf{v}) \\
f(\mathbf{u}) \leq f(\mathbf{v}) & \text { iff } \quad \forall i \in 1,2, \ldots, k: & f_{i}(\mathbf{u}) \leq f_{i}(\mathbf{v}) \\
f(\mathbf{u})<f(\mathbf{v}) & \text { iff } \quad f(\mathbf{u}) \leq f(\mathbf{v}) \wedge f(\mathbf{u}) \neq f(\mathbf{v})
\end{array}
$$

Then, in a minimization context, they comply with one of three conditions: 


$$
\begin{array}{lll}
\mathbf{u} \succ \mathbf{v} & (\mathbf{u} \text { dominates } \mathbf{v}), \text { iff } f(\mathbf{u})<f(\mathbf{v}) \\
\mathbf{v} \succ \mathbf{u} & (\mathbf{v} \text { dominates } \mathbf{u}), \text { iff } f(\mathbf{v})<f(\mathbf{u}) \\
\mathbf{u} \sim \mathbf{v} & (\mathbf{u} \text { and } \mathbf{v} \text { are non-comparable }), \text { iff } \mathbf{u} \nsucc \mathbf{v} \wedge \mathbf{v} \nsucc \mathbf{u}
\end{array}
$$

Alternatively, $\mathbf{u} \triangleright \mathbf{v}$ will denote that $\mathbf{u} \succ \mathbf{v}$ or $\mathbf{u} \sim \mathbf{v}$. A decision vector $\mathbf{x} \in$ $\mathbf{X}_{f}$ is non-dominated with respect to a set $V \subseteq \mathbf{X}_{f}$ iff: $\mathbf{x} \triangleright \mathbf{v}, \quad \forall \mathbf{v} \in V$. When $\mathbf{x}$ is non-dominated with respect to the whole set $\mathbf{X}_{f}$, it is called an optimal Pareto solution; therefore, the Pareto optimal set $\mathbf{X}_{\text {true }}$ may be formally defined as: $\mathbf{X}_{\text {true }}=\left\{\mathbf{x} \in \mathbf{X}_{f}: \mathbf{X}\right.$ is non-dominated with respect to $\left.\mathbf{X}_{f}\right\}$. The corresponding set of objective vectors $\mathbf{Y}_{\text {true }}=f\left(\mathbf{X}_{\text {true }}\right)$ constitutes the Optimal Pareto Front.

A solution $\mathbf{z}$ is attainable if there exists a solution $\mathbf{x} \in \mathbf{X}_{f}$ such that $\mathbf{z}=$ $f(\mathbf{x})$. The set of all attainable solutions is denoted as $\mathbf{Z}$. The ideal solution $\mathbf{z}^{*}$, is defined as $\mathbf{z}^{*}=\left(\min f_{1}(\mathbf{x}), \ldots, \min f_{k}(\mathbf{x})\right)$.

\section{The Multi-Objective TSP}

Given a complete, weighted graph $G=(N, E, d)$ with $N$ being the set of nodes, $E$ being the set of edges fully connecting the nodes, and $d$ being a function that assigns to each edge $\langle i, j\rangle \in E$ a vector $d_{i j}$, where each element corresponds to a certain measure (e.g. distance, cost) between $i$ and $j$, then the multi-objective TSP (MOTSP) [8] is the problem of finding a "minimal" Hamiltonian circuit of the graph, i.e., a closed tour visiting each of the $n=|N|$ nodes of $G$ exactly once, where "minimal" refers to the notion of Pareto optimality [8]. In this study, we consider symmetric problems, i.e. $d_{i j}=d_{j i}$ for all pairs of nodes $i, j$.

We will consider the bi-objective TSP:

$$
\begin{array}{ll}
\text { Minimize } & \mathbf{y}=f(\mathbf{x})=\left(y_{1}=f_{1}(\mathbf{x}), y_{2}=f_{2}(\mathbf{x})\right) \\
\text { subject to } & f(\mathbf{x})>0 \\
\text { where } & \mathbf{x}=(\langle 1,2\rangle,\langle 2,3\rangle, \ldots,\langle n-1, n\rangle,\langle n, 1\rangle) \in \mathbf{X} \\
\text { and } & \mathbf{y}=\left(y_{1}, y_{2}\right)=\left(f_{1}(\mathbf{x}), f_{2}(\mathbf{x})\right) \in \mathbf{Y}
\end{array}
$$

where $f_{1}$ and $f_{2}$ could be considered as the length of the tour, and the time required to traverse it respectively.

We will measure similarity of two solutions $\mathbf{x}, \mathbf{x}^{\prime}$ by the number of common edges $\langle i, j\rangle \in \mathbf{x}, \mathbf{x}^{\prime}$. On the contrary, the distance $\delta\left(\mathbf{x}, \mathbf{x}^{\prime}\right)$ is defined as the number of non-common edges, i.e. $n$ minus the similarity.

\section{Global Convexity}

The structure of the single-objective TSP has been studied by Boese et al. $[3,4]$. Their results indicate that the cost surface exhibits a globally convex structure, where good solutions are together in a small region of the search space, and the best solutions are located centrally with respect to the others. 
In a minimization context, Boese suggested an analogy with a big valley structure, in which the set of local minima appears convex with one central global minimum [4]. Even though there is no standard definition of global convexity, figure 1 gives an intuitive picture of a globally convex structure.

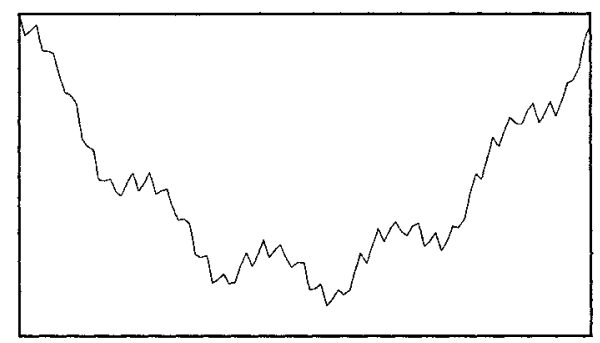

Fig. 1. Intuitive picture of the big valley or globally convex solution space structure

The global convexity idea is based on two assumptions [2]:

- Convexity: Local optima are gathered in a relatively small region of the solution space.

- Centrality: The best local optima are located centrally with respect to the population of local optima.

If both assumptions are valid, we should also expect that local optima are gathered in a small region close to the best local optimum [2]. Besides, any assessment of global convexity only makes sense once a topology has been established in the solution space [2].

Global Convexity has also been studied by Borges and Hansen in [2] for the three-objective TSP, by means of scalarization functions. These results were based on observed behavior rather than on theoretical analysis, and they are not very practical. In fact, Borges and Hansen reduced the multi-objective problem to a single-objective one [2], loosing several characteristics of a truly multiobjective problem, whose theoretical solution is a whole Pareto set and not an ideal solution which is not attainable in practice. Therefore, this work introduces truly multi-objective concepts as category and range, trying to achieve a more general multi-objective framework. This generalization allows a more rigorous analysis of a MOP for any number of objective functions or measurement units.

\section{Topological Analysis of the Solution Space}

Boese used the length of a tour to study the quality of a solution [4], what is completely valid in a single-objective context. For a MOP, Borges and Hansen proposed the use of scalarization functions that reduce the multi-objective problem to a single-objective one [2]. 
In what follows, the concepts of category and range of a solution are presented for the first time as quality metrics, to allow a further topological analysis of the bi-objective TSP.

A population $P=\left\{\mathbf{x}_{1}, \mathbf{x}_{2}, \ldots, \mathbf{x}_{|P|}\right\}$ is defined as a set of valid solutions $\mathbf{x}_{i} \in \mathbf{X}_{f}$ of the bi-objective TSP, with cardinality $|P|$.

Definition 1. Let $P \subset \mathbf{X}_{f}$ be a population, and $\mathbf{x} \in P$ a solution. The category of a solution $\mathbf{x}$ in a population $P$ is defined as:

$$
\operatorname{cat}(\mathbf{x}, P)=|\{\mathbf{u} \in P: \mathbf{u} \succ \mathbf{x}\}|
$$

Then, the category of the solution $\mathrm{x}$ is the number of solutions in $P$ that dominates $\mathbf{x}$. Therefore, a solution of the Pareto front will always have a 0 category, i.e. if $\mathbf{u} \in \mathbf{X}_{\text {true }}$ then $\operatorname{cat}\left(\mathbf{u}, \mathbf{X}_{f}\right)=0$.

Definition 2. Let $P \subset \mathbf{X}_{f}$ be a population. The non-dominated frontier of $P$ is defined as:

$$
\mathbf{N F}(P)=\{\mathbf{u} \in P: \operatorname{cat}(\mathbf{u}, P)=0\}
$$

If $P=\mathbf{X}_{f}$ then $\mathbf{N F}(P)=\mathbf{X}_{\text {true }}$.

Definition 3. Let $P \subset \mathbf{X}_{f}$ be a population, and $\mathbf{x} \in P$ a solution. The range of a solution $\mathbf{x}$ in a population $P$, denoted as $r n g(\mathbf{x}, P)$, is defined according to the following algorithm:

$$
\begin{aligned}
& \text { if } \mathbf{x} \in \mathbf{N F}(P) \text { then } \operatorname{rng}(\mathbf{x}, P)=0 \\
& \text { else } \quad r n g(\mathbf{x}, P)=1+r n g\left(\mathbf{x}, P^{\prime}\right) \text { where } P^{\prime}=P-\mathbf{N F}(P)
\end{aligned}
$$

From now on, the use of the parameter $P$ will be omitted from the range and category notation. Therefore, they will be denoted as $r n g(\mathbf{x})$ and $\operatorname{cat}(\mathbf{x})$ respectively. The parameter $P$ is left only for ambiguous cases.

A definition of distance is now presented for the study of global convexity in the bi-objective TSP.

Definition 4. Let $P \subset \mathbf{X}_{f}$ be a population, and $\mathbf{x} \in P$ a solution. The mean distance of a solution $\mathbf{x}$ to a population $P$ is defined as:

$$
\delta(\mathbf{x}, P)=\frac{1}{|P|-1} \sum_{i=1}^{|P|} \delta(\mathbf{u}, \mathbf{x}) \quad \forall \mathbf{u} \in P .
$$

This paper is inspired in Boese's approach [4], where different solutions of an $n$ city problem are saved in a set $P$; consequently, each solution $\mathrm{x}$ has:

- A category cat $(\mathbf{x})$.

- A range $r n g(\mathbf{x})$.

- A mean distance to the other solutions of $P$ denoted as $\delta(\mathrm{x}, P)$.

- A distance to the non-dominated frontier denoted as $\delta(\mathbf{x}, \mathbf{N F}(P)$ ) (defined in the next section). 
This work is divided in two parts. For the first part, small random instances were thoroughly analyzed, and for the second part, analyses based on larger instances from TSPLIB ${ }^{1}$ were made.

\subsection{Exhaustive Study of the Solution Space}

The study is based on random generated instances with $7,8,9$ and 10 cities, named litAB7, omiAB8, encAB9 and asuAB10. These problems are described in [9].

Due to the presence of multiple optimal solutions, a definition of distance to the non-dominated frontier is needed.

Definition 5. Let $P \subset \mathbf{X}_{f}$ be a population, and $\mathbf{x} \in P$ a solution. The distance of a solution $\mathrm{x}$ to the non-dominated frontier of $P$ is defined as:

$$
\delta(\mathbf{x}, \mathbf{N F}(P))=\min \left\{\delta\left(\mathbf{x}, \mathbf{x}_{i}^{*}\right): \mathbf{x}_{i}^{*} \in \mathbf{N F}(P)\right\}
$$

The $e$ best solutions of $P$ will be denoted as $P_{(e)}$; e.g. $P_{(100)}$ denotes the set of the best 100 solutions of $P$, i.e., the 100 solutions with the smallest category.

For the calculations, an exhaustive search was made. The obtained population is the whole solution space for an $n$ city problem, i.e. $P=\mathrm{X}_{f}$, therefore, $\left|\mathbf{X}_{f}\right|=|P|=\frac{(n-1) !}{2}$, and, $\mathbf{N F}(P)=\mathbf{X}_{\text {true }}$.

For each solution $\mathbf{x} \in P$, correlations between the following variables were calculated:

- the distance to the non-dominated frontier $\delta(\mathbf{x}, \mathbf{N F}(P))$ and the category of a solution $\operatorname{cat}(\mathbf{x})$, denoted as $\rho(\operatorname{cat}(\mathbf{x}), \delta(\mathbf{x}, \mathbf{N F}(P)))$;

- the mean distance to the population $\delta(\mathrm{x}, P)$ and the category of a solution $\operatorname{cat}(\mathbf{x})$ denoted as $\rho(\operatorname{cat}(\mathbf{x}), \delta(\mathbf{x}, P))$;

- the distance to the non-dominated frontier $\delta(\mathrm{x}, \mathrm{NF}(P))$ and the mean distance to the population $\delta(\mathbf{x}, P)$ denoted as $\rho(\delta(\mathbf{x}, P), \delta(\mathbf{x}, \mathbf{N F}(P)))$;

- the mean distance to the population $\delta(\mathrm{x}, P)$ and the range of a solution $r n g(\mathbf{x})$ denoted as $\rho(\operatorname{rng}(\mathbf{x}), \delta(\mathbf{x}, P))$;

- the distance to the non-dominated frontier $\delta(\mathbf{x}, \mathbf{N F}(P))$ and the range of a solution $r n g(\mathbf{x})$ denoted as $\rho(r n g(\mathbf{x}), \delta(\mathbf{x}, \mathbf{N F}(P)))$;

- the range and category of a solution denoted as $\rho(\operatorname{rng}(\mathbf{x})$, cat $(\mathbf{x}))$.

A summary for these values is shown in tables 1 to 4 , and the figures for these correlations can be found in [9].

These results suggest that range and category are very similar quality metrics, with correlations between them larger than 0.9 .

High values can be observed for the correlations $\rho(\operatorname{cat}(\mathbf{x}), \delta(\mathbf{x}, P))$ and $\rho(r n g(\mathbf{x}), \delta(\mathbf{x}, P))$, which suggests a concentration of very good solutions in the center of the solution space, satisfying the centrality assumption of a globally convex structure. Also, the correlations $\rho(\operatorname{cat}(\mathbf{x}), \delta(\mathbf{x}, \mathbf{N F}(P)))$ and

\footnotetext{
${ }^{1}$ http://www.iwr.uni-heidelberg.de/groups/comopt/software/TSPLIB95/
} 
Table 1. Correlations for the problem litAB7

\begin{tabular}{l|cccc}
\hline & $\bar{P}$ & $\bar{P}_{(|P|-1)}$ & $P_{\left(\frac{|P|}{2}\right)}$ & $P_{\left(\frac{|P|}{4}\right)}$ \\
\hline$\rho(\operatorname{cat}(\mathbf{x}), \delta(\mathbf{x}, P))$ & 0 & 0.602793 & 0.761135 & 0.659006 \\
$\rho(\operatorname{cat}(\mathbf{x}), \delta(\mathbf{x}, \mathbf{N F}(P)))$ & 0.767441 & 0.763554 & 0.548112 & 0.585267 \\
$\rho(\delta(\mathbf{x}, P), \delta(\mathbf{x}, \mathbf{N F}(P)))$ & 0 & 0.582333 & 0.633253 & 0.706702 \\
$\rho(r n g(\mathbf{x}), \delta(\mathbf{x}, P))$ & 0 & 0.628991 & 0.752562 & 0.661246 \\
$\rho(r n g(\mathbf{x}), \delta(\mathbf{x}, \mathbf{N F}(P)))$ & 0.786593 & 0.783295 & 0.567543 & 0.633579 \\
$\rho(r n g(\mathbf{x}), \operatorname{cat}(\mathbf{x}))$ & 0.960048 & 0.960550 & 0.937163 & 0.933828 \\
\hline
\end{tabular}

Table 2. Correlations for the problem omiAB8

\begin{tabular}{l|cccc}
\hline & $P$ & $P_{(|P|-1)}$ & $P\left(\frac{|P|}{2}\right)$ & $P\left(\frac{|P|}{4}\right)$ \\
\hline$\rho(\operatorname{cat}(\mathbf{x}), \delta(\mathbf{x}, P))$ & 0 & 0.645277 & 0.868166 & 0.782142 \\
$\rho(\operatorname{cat}(\mathbf{x}), \delta(\mathbf{x}, \mathbf{N F}(P)))$ & 0.774954 & 0.774225 & 0.627201 & 0.524996 \\
$\rho(\delta(\mathbf{x}, P), \delta(\mathbf{x}, \mathbf{N F}(P)))$ & 0 & 0.549804 & 0.686872 & 0.555586 \\
$\rho(r n g(\mathbf{x}), \delta(\mathbf{x}, P))$ & 0 & 0.644026 & 0.929499 & 0.853578 \\
$\rho(r n g(\mathbf{x}), \delta(\mathbf{x}, \mathbf{N F}(P)))$ & 0.810660 & 0.810222 & 0.678969 & 0.594514 \\
$\rho(r n g(\mathbf{x}), \operatorname{cat}(\mathbf{x}))$ & 0.976814 & 0.976843 & 0.973864 & 0.959215 \\
\hline
\end{tabular}

Table 3. Correlations for the problem encAB9

\begin{tabular}{l|cccc}
\hline & $P$ & $P(|P|-1)$ & $P\left(\frac{|P|}{2}\right)$ & $P\left(\frac{|P|}{4}\right)$ \\
\hline$\rho(\operatorname{cat}(\mathbf{x}), \delta(\mathbf{x}, P))$ & 0 & 0.572255 & 0.825523 & 0.697679 \\
$\rho(\operatorname{cat}(\mathbf{x}), \delta(\mathbf{x}, \mathbf{N F}(P)))$ & 0.641862 & 0.641802 & 0.435708 & 0.409532 \\
$\rho(\delta(\mathbf{x}, P), \delta(\mathbf{x}, \mathbf{N F}(P)))$ & 0 & 0.329447 & 0.478431 & 0.371982 \\
$\rho(\operatorname{rng}(\mathbf{x}), \delta(\mathbf{x}, P))$ & 0 & 0.564819 & 0.894474 & 0.759983 \\
$\rho(r n g(\mathbf{x}), \delta(\mathbf{x}, \mathbf{N F}(P)))$ & 0.672059 & 0.671998 & 0.483163 & 0.492397 \\
$\rho(\operatorname{rng}(\mathbf{x}), \operatorname{cat}(\mathbf{x}))$ & 0.971607 & 0.971621 & 0.974710 & 0.971266 \\
\hline
\end{tabular}

Table 4. Correlations for the problem asuAB10

\begin{tabular}{l|cccc}
\hline & $P$ & $P(|P|-1)$ & $P\left(\frac{|P|}{2}\right)$ & $P\left(\frac{|P|}{4}\right)$ \\
\hline$\rho(\operatorname{cat}(\mathbf{x}), \delta(\mathbf{x}, P))$ & 0 & 0.508309 & 0.830280 & 0.734061 \\
$\rho(\operatorname{cat}(\mathbf{x}), \delta(\mathbf{x}, \mathbf{N F}(P)))$ & 0.712957 & 0.712944 & 0.536178 & 0.488927 \\
$\rho(\delta(\mathbf{x}, P), \delta(\mathbf{x}, \mathbf{N F}(P)))$ & 0 & 0.392366 & 0.577878 & 0.442818 \\
$\rho(\operatorname{rng}(\mathbf{x}), \delta(\mathbf{x}, P))$ & 0 & 0.493534 & 0.899061 & 0.803484 \\
$\rho(r n g(\mathbf{x}), \delta(\mathbf{x}, \mathbf{N F}(P)))$ & 0.750817 & 0.750809 & 0.583105 & 0.533937 \\
$\rho(\operatorname{rng}(\mathbf{x}), \operatorname{cat}(\mathbf{x}))$ & 0.970935 & 0.970937 & 0.976167 & 0.975501 \\
\hline
\end{tabular}

$\rho(r n g(\mathbf{x}), \delta(\mathrm{x}, \mathbf{N F}(P)))$, indicate that these solutions are gathered in a relatively small region of the solution space, satisfying the convexity assumption.

As both assumptions are fulfilled, it is expected that these solutions are close to the Pareto front, which is consistent with a globally convex structure conjecture.

The correlations for the bi-objective TSP do not present the high values obtained by Boese for the single-objective case [4]. The reason for this fact is due to the existence of a whole set of Pareto solutions. As a consequence, non- 
Pareto solutions could be more central in the solution space than other Pareto solutions.

Larger problems are analyzed in the next section using the same metrics with subsets of the solution space.

\subsection{Study of a Subset of Solutions}

This analysis was based on the TSPLIB instances kroAB100, kroCD100, kroAD100, kroBC100 and kroAB150. From each of these bi-objective TSPs, $n^{2}$ random samples were taken. The local search algorithm 2-Opt was used for the optimization of each sample set, achieving populations containing local optima solutions. This search strategy was chosen because it presents a simple neighborhood structure, and allows the study of local optima distribution in the solution space.

The methodology of the analysis remains the same, using subsets of the solution space instead of considering the whole solution space. The correlations obtained are shown in table 5 .

Table 5. Correlations for the instances kroAB100, kroCD100, kroAD100, kroBC100, kroAB150

\begin{tabular}{l|lllll}
\hline & kroAB100 kroCD100 kroAD100 & kroBC100 kroAB150 \\
\hline$\rho($ cat $(\mathbf{x}), \delta(\mathbf{x}, P))$ & 0.616596 & 0.596558 & 0.603098 & 0.589122 & 0.571318 \\
$\rho(\operatorname{cat}(\mathbf{x}), \delta(\mathbf{x}, \mathbf{N F}(P)))$ & 0.396173 & 0.401700 & 0.391263 & 0.396632 & 0.384648 \\
$\rho(\delta(\mathbf{x}, P), \delta(\mathbf{x}, \mathbf{N F}(P)))$ & 0.613169 & 0.626019 & 0.611728 & 0.653360 & 0.633580 \\
$\rho($ rng $(\mathbf{x}), \delta(\mathbf{x}, P))$ & 0.639641 & 0.628951 & 0.645090 & 0.617764 & 0.606404 \\
$\rho(\operatorname{rng}(\mathbf{x}), \delta(\mathbf{x}, \mathbf{N F}(P)))$ & 0.432760 & 0.441982 & 0.449073 & 0.437721 & 0.423266 \\
$\rho($ rng $(\mathbf{x})$, cat $(\mathbf{x}))$ & 0.945914 & 0.941865 & 0.937078 & 0.941890 & 0.938438 \\
\hline
\end{tabular}

The results for the problem kroAB100 are shown in figure 2. The figures for the other problems can be found in [9]. Correlations between range and category, still maintain a value larger than 0.9 , which confirms their similarity, although range presents better results in the whole study.

A concentration of the best solutions centrally with respect to the population is observed in figures 2.a and 2.d. Despite low correlations in the previous figures (around 0.6), figure 2.c shows that solutions located centrally are closer to the non-dominated frontier, and suggests the existence of a globally convex structure. The same results were obtained for the rest of the studied instances.

\section{Conclusions and Future Work}

The concepts of category and range proved to be very effective quality metrics for the bi-objective TSP, and the generalization of these concepts can be easily made for any instance of the MOTSP. Besides, they can be used in any MOP 


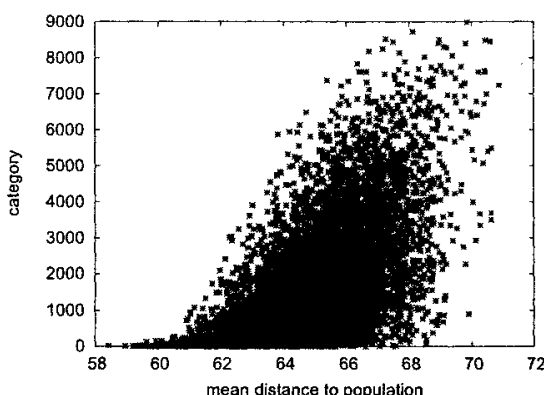

(a) $\rho(\operatorname{cat}(\mathbf{x}), \delta(\mathbf{x}, P))=0.616596$

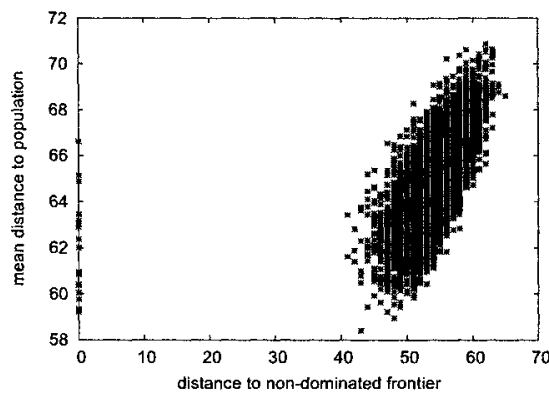

(c) $\rho(\delta(\mathbf{x}, P), \delta(\mathbf{x}, \mathbf{N F}(P)))=0.613169$

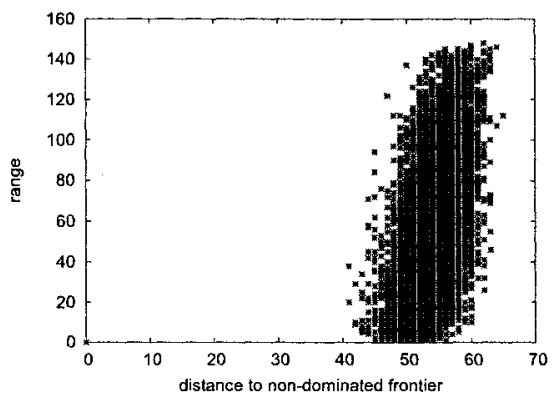

(e) $\rho(r n g(\mathbf{x}), \delta(\mathbf{x}, \mathbf{N F}(P)))=0.432760$

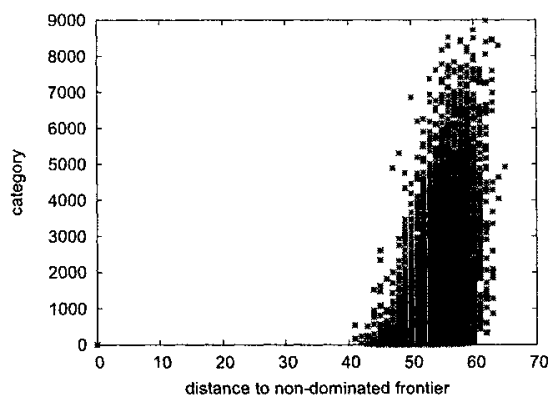

(b) $\rho(\operatorname{cat}(\mathbf{x}), \delta(\mathbf{x}, \mathbf{N F}(P)))=0.396173$

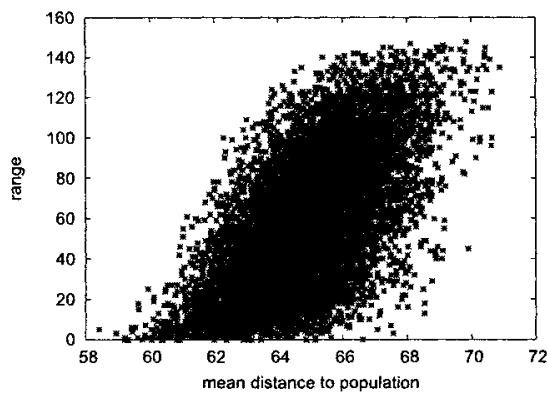

(d) $\rho(r n g(\mathbf{x}), \delta(\mathbf{x}, P))=0.639641$

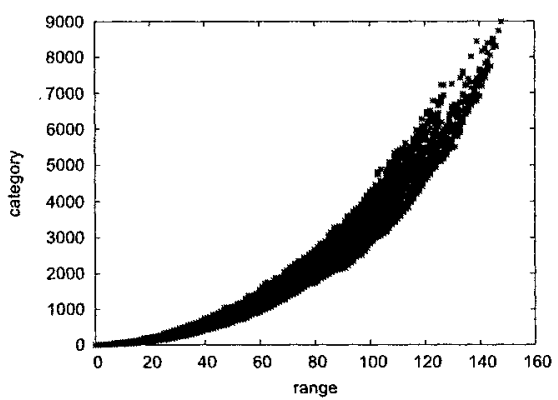

(f) $\rho(\operatorname{cat}(\mathbf{x}), \operatorname{rng}(\mathbf{x}))=0.945914$

Fig. 2. Population of 10,000 optimized solutions for the instance kroAB100

without definition changes. Although these concepts seem to be very similar, range showed better results.

Category, range, mean distance to the population and distance to the nondominated frontier experimentally demonstrated to be correlated, showing the topological characteristic of global convexity. These metrics could be used for the study of global convexity in other MOPs, where metaheuristics, as ACO or EA, have shown to be very efficient, and for the creation of new metaheuris- 
tics that could exploit this type of structure. However, since the results were obtained experimentally, it is not certain that this structure holds for every instance of the bi-objective TSP. Nevertheless, for the single-objective case, no instance was found without a globally convex structure [6].

A problem with a known global convexity structure will allow us to limit the search to a smaller solution area, and from there, it will be possible to use another appropriate algorithms to achieve better approximations to the Pareto set.

There is a lot to do in the study of global convexity, like the creation of metaheuristics based on the exploitation of this structure, the study of instances with correlated objectives, and the use of another kind of neighborhood structure (different than 2-Opt). Also, it can be considered the development of a formal theory for global convexity, and the identification of globally convex problems. Just $[3,4,6]$ refers to the subject of global convexity in the TSP, and [2] for the MOTSP.

\section{References}

1. C. Blum, and A. Roli, Metaheuristics in Combinatorial Optimization: Overview and Conceptual Comparison, ACM Computing Surveys (CSUR), 35(3), 268-308 (2003).

2. P.C. Borges, and P.H. Hansen, A Study of Global Convexity for a Multiple Objective Traveling Salesman Problem, edited by C.C. Ribeiro and P. Hansen, (Essays and Surveys in Metaheuristics, Kluwer, 2000), pp. 129-150.

3. K. Boese, A. Kahng, and S. Muddu, A new Adaptive Multi-Start Technique for Combinatorial Global Optimization, Operations Research Letters, 16, 101-113 (1994).

4. K. Boese, Cost versus Distance in the Traveling Salesman Problem, Computer Science Department, University of California, Technical Report No. 950018, 1995.

5. T. Stützle, and H.H. Hoos, Max-Min Ant System, Future Generation Computer Systems, 16(8), 889-914 (2000).

6. B. Barán, P. Gardel, and O. Gómez, Estudio del Espacio de Soluciones del Problema del Cajero Viajante, edited by M. Solar, D. Fernández and E. CuadrosVargas, (CLEI 2004, Arequipa, Perú, 2004), pp. 745-756.

7. O. Gómez, and B. Barán, Reasons of ACO's Success in TSP, edited by M. Dorigo, M. Birattari, C. Blum, L. Gambardella, F. Mondada and T. Stüzle, (Ant Colony Optimization and Swarm Intelligence, 4th International Workshop, ANTS 2004), pp. 226-237.

8. L. Paquete, M. Chiarandini, and T. Stützle, A Study of Local Optima in the Biobjective Travelling Salesman Problem, Computer Science Department, Darmstadt University of Technology, Technical Report No. AIDA-02-07, 2002 (Presented at the Multi-Objective Metaheuristics Workshop, MOMH 2002).

9. M. Villagra, B. Barán, and O. Gómez, Convexidad Global en el Problema del Cajero Viajante Bi-Objetivo, Centro Nacional de Computación, Universidad Nacional de Asunción, Technical Report No. 001/05, 2005 (unpublished); http://www.cnc.una.py. 